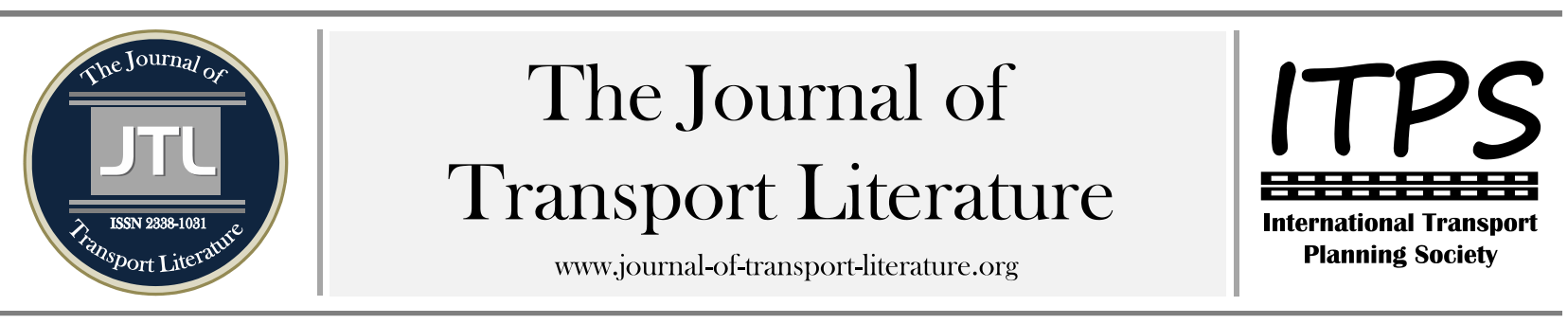

\title{
Procedimento de escolha e análise de alternativas de transporte para exportação de bioetanol no Brasil
}

Yuka Akasaka ${ }^{1}$; Marcelino Aurélio Vieira da Silva ${ }^{1,+}$; Ilton Curty Leal Jr²

${ }^{1}$ Federal University of Rio de Janeiro, Rio de Janeiro, Brazil

${ }^{2}$ Federal Fluminense University, Niterói, Brazil

\section{Article Info}

Palavras-chave:

lógica fuzzy

bioetanol

escolha modal

Submitted 10 Feb 2014;

received in revised form 2 Sep 2014;

accepted 13 Oct 2014

Licensed under

Creative Commons

CC-BY 3.0 BR

\section{Resumo}

O presente trabalho apresenta um procedimento para auxiliar a análise de desempenho de alternativas de transportes com o emprego combinado de variáveis quantitativas e linguísticas, diferenciando-se das avaliações tradicionais que utilizam em suas aplicações variáveis com comportamento quantitativo. 0 procedimento proposto utiliza como ferramenta a lógica fuzzy para modelar as variáveis linguísticas que representam o nível de serviço das alternativas e os pesos entre os atributos do problema. 0 procedimento foi aplicado para o caso de transporte de bioetanol para o qual se avaliaram as categorias de custo e de nível de serviço de alternativas multimodais pela perspectiva dos especialistas da área. Após isso, realizou-se análise de sensibilidade por meio de cenários com variações de importância destas categorias. Os resultados mostram que na avaliação dos especialistas há uma tendência em se atribuir uma maior importância ao nível de serviço em comparação ao custo da operação e que a prática atual de transporte não é a alternativa mais adequada considerando o ponto de vista dos especialistas.

+ Corresponding author. Universidade Federal do Rio de Janeiro. Cidade Universitária. 21949900 - Rio de Janeiro, RJ - Brasil.

E-mail address: marcelino@pet.coppe.ufrj.br.

\section{Introdução}

Este trabalho visa desenvolver um procedimento de análise de desempenho de alternativas em relação ao custo e nível de serviço. Devido às características qualitativas presentes nos atributos necessários para avaliar o nível de serviço, definiuse como hipótese que seria possível utilizar a lógica Fuzzy com emprego de variáveis linguísticas para modelar a análise de desempenho estudada. Como premissa do trabalho, definiu-se que é suficiente avaliar as alternativas multimodais da aplicação em função do nível de servido e do custo. No estudo realizado por Leal Jr. e D’Agosto (2011a) é apresentado uma visão paradoxal ao cenário atual da rota de exportação do bioetanol com destinação ao porto. Foram avaliadas, por meio de análise de desempento, alternativas utilizando a intermodalidade entre os modos rodoviário, aquaviário, dutoviário e ferroviário com terminais de transbordo ao longo do percurso, e verificou-se que na análise dos aspectos financeiros e socioambientais, o cenário onde apresentou melhor resultado foi a alternativa onde envolve o sistema de dutos em grande escala. As alternativas com os índices menos eficientes e mais custosos foram onde houve utilização representativa do modelo atual de transporte de bioetanol, o modo rodoviário.

A avaliação de desempenho pode ser empregada para diferentes finalidades, a seguir serão detalhados alguns estudos desta natureza. Lieggio Junior et al (2012) apresenta um estudo de aplicação de técnicas multicritério para avaliar atividade de transporte de produtos perigosos. Leal Jr \& D'Agosto (2011b) apresenta um método de escolha modal com base em medidas de ecoeficiência. Guabiroba et al (2014) apresenta um método de definição de consórcios de coleta de lixo com base em medidas de ecoeficiência. Bertolo et al (2011), Falcão \& Correia (2012) e Acosta et al (2011) aplicaram a análise de desempenho com a finalidade de avaliar a eficiência de portos brasileiros. Cortez et al (2013) aplicou a análise de desempenho para avaliar a eficiência na gestão de portos brasileiros. Gabriele et al (2013) Comparou a eficiência ambiental dos modos de transporte rodoviário e ferroviário. Ciarlini e Correia (2011) avaliaram o sistema de transporte rápido de massa. Fernandes e Pires (2011) avaliaram o desempenho de empresas aéreas. Em relação ao transporte de bioetanol, surge o questionamento do motivo pelo qual o modelo logístico atual é o utilizado mesmo com todas as suas desvantagens apresentadas anteriormente. Para tanto, serão apresentados neste trabalho a avaliação em termos de custo e de nível de serviço em cada alternativa de intermodalidade expostas no estudo de Leal Jr \& D’Agosto (2011a). Baseada na lógica Fuzzy, os desempenhos destas categorias e seus atributos foram avaliados dispondo de questionários aos especialistas da área, onde as variáveis linguísticas foram transformadas em modelos quantitativos.

A partir desta introdução, o trabalho está dividido em quatro seções, sendo que a primeira seção trata do referencial teórico onde a avaliação de desempenho no transporte e a lógica Fuzzy são abordadas. Na segunda e na terceira seções estão sendo detalhados respectivamente a metodologia deste trabalho e o desenvolvimento do estudo de caso. A quarta e última parte aborda a conclusão e considerações finais deste artigo. 


\section{Metodologia}

A Figura 1 apresenta o procedimento proposto para avaliar as alternativas modais, considerando a existências de variáveis qualitativas. 0 procedimento foi elaborado baseado no trabalho de Liang e Wang (1991), realizando as adequações necessárias ao problema deste artigo.

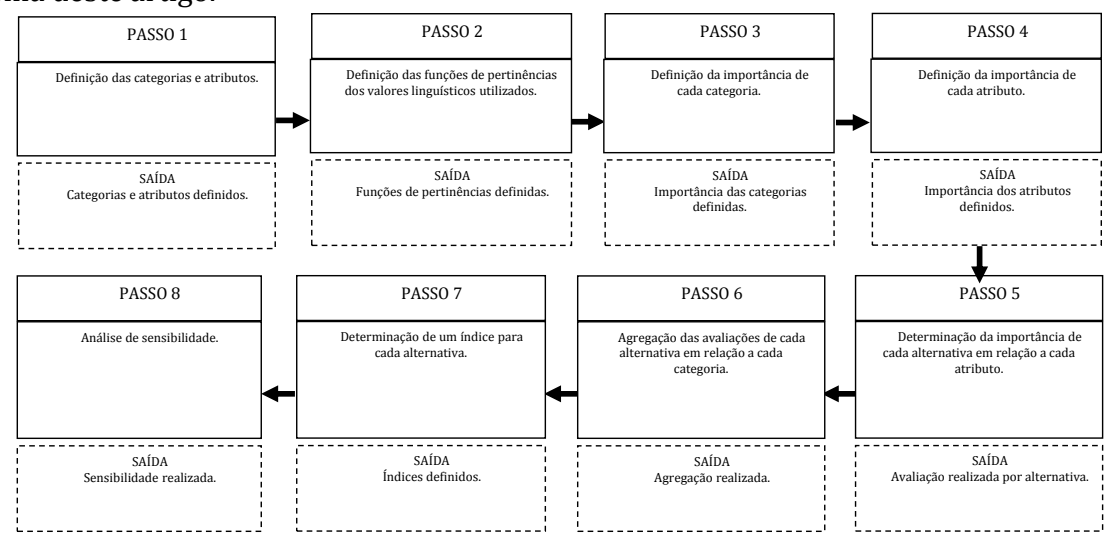

Figura 1 - Procedimento proposto (fonte: autores).

\section{Estudo de caso}

\subsection{Definição dos critérios}

Para o presente trabalho foram definidas duas categorias: Custo e Nível de Serviço. Em relação ao custo foi avaliado o atributo do valor do frete total. Para o nível de serviço foram avaliados os atributos: Segurança, Flexibilidade, Tempo, Capacidade e Confiabilidade. A Figura 2 mostra a árvore de critérios para o caso estudado.

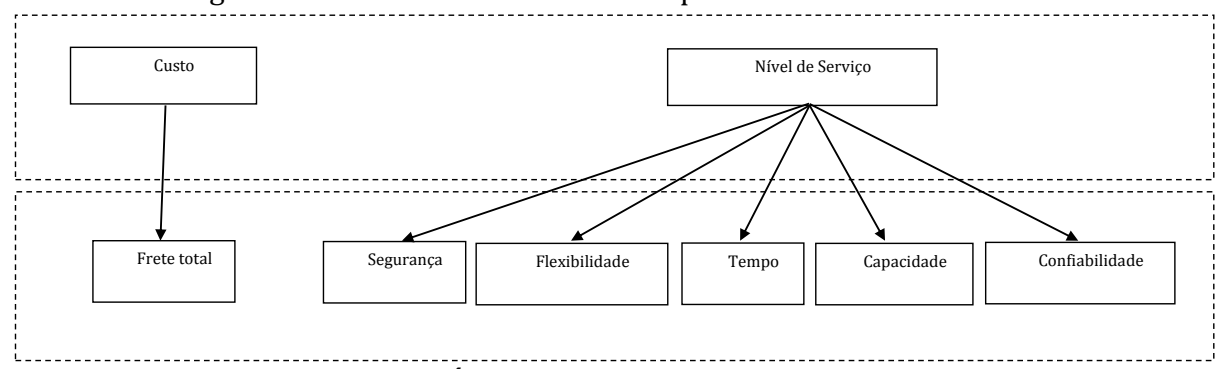

Figura 2 - Árvore de atributos (fonte: autores).

0 atributo custo é um dos mais citados pela literatura e normalmente considerado um dos mais importantes no grupo dos econômico-financeiros. Na visão do transportador, os custos correspondem ao custo médio de transporte (fixos e variáveis) mais as taxas adicionais (impostos, pedágios, seguros e etc). Pela visão do embarcador, os custos são os valores de frete, que representam a receita recebida pelos transportadores (Leal Jr., 2010). Para o presente trabalho a visão a ser utilizado é do embarcador e o atributo é o frete total. Dentre a categoria de nível de serviço, foram definidos cinco atributos analisados nas perspectivas de Leal Jr (2010) como pode ser visto na Figura 2. A Figura 3 mostra as 10 alternativas (A1 a A10 - multimodais ou unimodais) para se transportar bioetanol de uma cidade do interior de Goiás, chamada Turvelândia, para o porto de São Sebastião em SP. As setas representam os modos de transporte, conforme legenda, e os círculos os terminais de transbordo. Para o caso de terminais unindo o mesmo modo de transporte não há transbordo.

\subsection{Definição das funções de pertinências para os valores linguísticos utilizados}

A coleta de informação foi realizada através da aplicação de questionários para especialistas do setor. Um grupo de três avaliadores definiu o grau de pertinência entre 0 a 1 para os valores linguísticos. Para a definição da função de pertinência foi utilizada uma média dos graus atribuídos. Trabalhos como de Liang e Wang (1991) retratam da mesma forma a utilização de poucos dados, porém confiáveis. Para avaliação da importância entre categorias e entre os atributos da categoria de nível de serviço foram utilizados os seguintes valores linguísticos: "Muito Pouco Importante" (MPI), "Pouco Importante" (PI), "Importante" (I), "Muito Importante" (MI) e "Importantíssimo" (II). E para avaliação da característica de cada atributo da categoria de nível de serviço em determinada alternativa multimodal, foram atrelados os seguintes valores linguísticos: "Baixíssimo" (MMB), "Muito baixo" (MB), "Baixo" (B), "Médio" (M), "Alto" (A), "Muito Alto" (MA) e "Altíssimo" (MMA). A representação triangular dos valores linguísticos podem ser visualizadas na Figura 4.

\subsection{Definição da importância de cada categoria}

Cada membro i do conjunto de especialista atribuiu um valor linguístico (Sij) para cada categoria j e as respostas estão apresentadas na Tabela 1. A importância de cada categoria j (Sj) é determinada pela Equação 1.

$$
S_{j}=\sum_{i=1}^{I} \frac{S_{i j}}{I} \text { para i }=1,2, \ldots \mathrm{I} ; \mathrm{j}=1,2
$$

em que I: é o número total de especialistas. Os valores de Sj, representados da forma de um número Fuzzy Sj (qj, oj, pj, rj), estão apresentados na Tabela 1. 


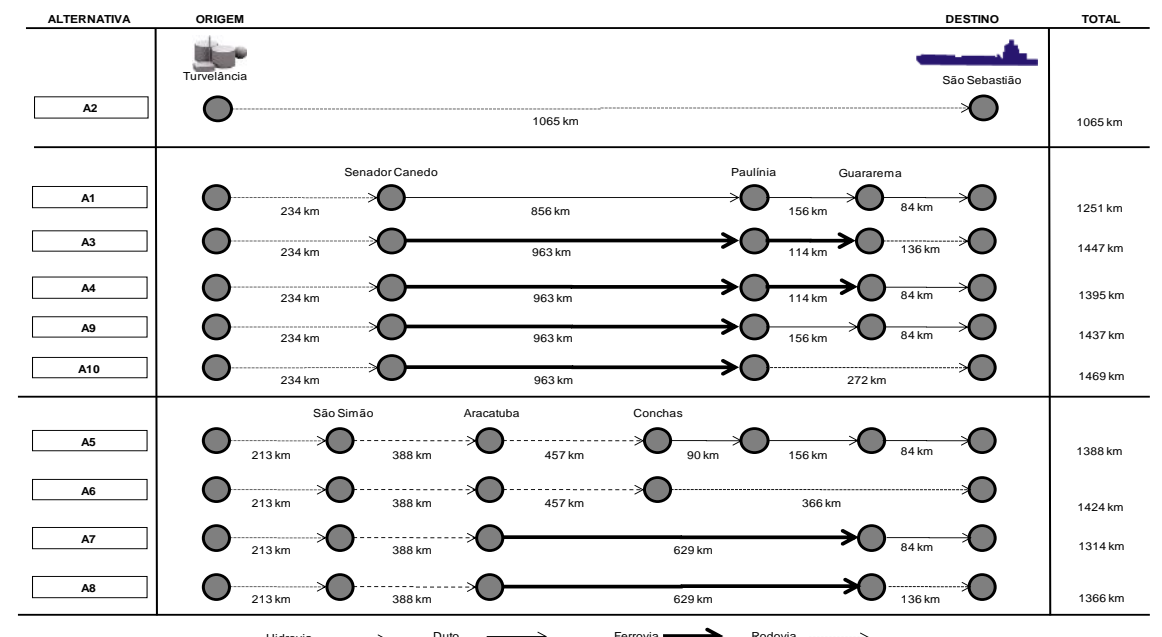

Figura 3 - Alternativas para transporte de bioetanol (fonte: Leal Jr. \& D'Agosto, 2011).

Notas: Veículos rodoviários bitrem tanque com capacidade para $45 \mathrm{~m} 3$. Composição ferroviária com 100 vagões-tanque com capacidade de $103 \mathrm{~m} 3$ cada. Comboio hidroviário com 4 chatas com capacidade de $1270 \mathrm{~m} 3$ cada. Alcoolduto com capacidade de $33.000 \mathrm{~m} 3 /$ dia.
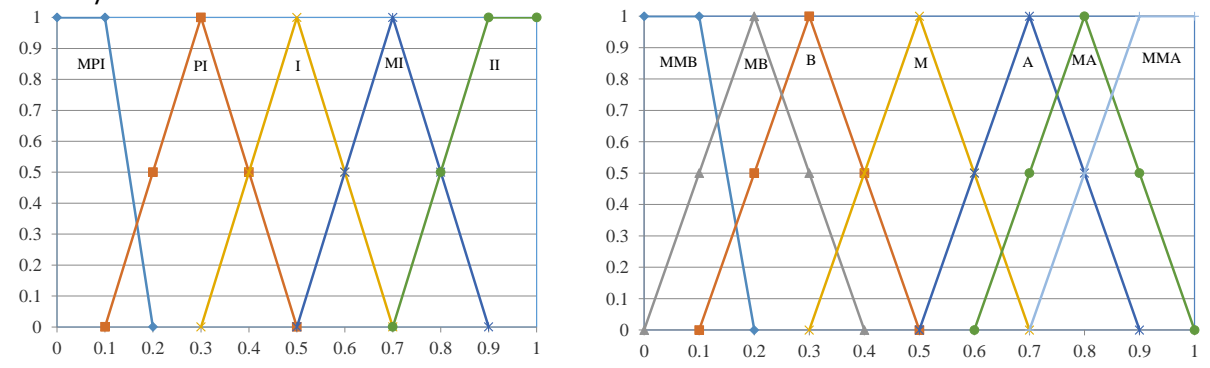

Figura 4 - Funções de pertinências (fonte: autores).

Tabela 1 - Avaliação dos especialistas e Importância para as categorias (fonte: autores).

\begin{tabular}{cccccccc}
\hline & \multicolumn{3}{c}{ Avaliação dos Especialistas } & \multicolumn{3}{c}{ Importância } \\
Categorias & Esp 1 & Esp 2 & Esp 3 & q & o & P & r \\
\hline Custo (j=1) & II & II & II & 0,70 & 0,9 & 1 & 1 \\
Nível de Serviço (j=2) & I & I & PI & 0,23 & 0,43 & 0,43 & 0,63 \\
\hline
\end{tabular}

\subsection{Definição da importância de cada atributo}

Nesta etapa será determinada a importância de cada atributo t (At) da categoria nível de serviço. Cada membro i do conjunto de especialista atribuiu um valor linguístico (Ait) para cada atributo t e as respostas estão apresentadas na Tabela 2. De forma similar a etapa anterior, a importância de cada atributo At é determinada pela média dos números Fuzzy conforme apresentado na Equação 1. Os valores de At, representados da forma de um número Fuzzy At (qt, ot, pt, rt), estão apresentados na Tabela 2 Como citado anteriormente à importância dos atributos é a mesma do aspecto correspondente.

Tabela 2 - Avaliação dos especialistas e Importância para os atributos (fonte: autores).

\begin{tabular}{cccccccc}
\hline \multicolumn{1}{c}{ Taliação dos Especialistas } & \multicolumn{5}{c}{ Importância } \\
Categorias & Esp 1 & Esp 2 & Esp 3 & q & o & P & r \\
\hline Segurança (t=1) & I & I & I & 0,30 & 0,50 & 0,50 & 0,70 \\
Flexibilidade (t=2) & PI & I & PI & 0,17 & 0,37 & 0,37 & 0,57 \\
Tempo (t=3) & II & II & MI & 0,63 & 0,83 & 0,90 & 0,97 \\
Capacidade (t=4) & II & I & MI & 0,50 & 0,70 & 0,73 & 0,87 \\
Confiabilidade (t=5) & I & I & II & 0,43 & 0,63 & 0,67 & 0,80 \\
\hline
\end{tabular}

\subsection{Definição da importância de cada alternativa em relação a cada atributo}

Buscando determinar a importância de cada alternativa $\mathrm{k}$ em relação a cada atributo da categoria de nível de serviço $\mathrm{t}$ (Alkt), foi calculada a média dos números Fuzzy que representam a avaliação qualitativa da importância dada por cada especialista i para cada alternativa k em relação a cada atributo t (AIkti).

Das respostas dos especialistas temos os seguintes números Fuzzy para o problema:

$$
A_{\text {lkti }}=\left(q_{k t i} o_{k t i} p_{k t i} r_{k t i}\right), k=1,2, \ldots 10 ; t=1,2, \ldots 5 \mathrm{e} \mathrm{i}=1,2 \mathrm{e} 3 \text {. }
$$

Em que k: alternativas multimodais; t: atributos do nível de serviço; i: especialistas. Logo, a importância de cada alternativa em relação a cada aspecto é dada por:

$$
\mathrm{A}_{\mathrm{lkt}}=\left(\mathrm{q}_{\mathrm{kt}} \mathrm{o}_{\mathrm{kt}} \mathrm{p}_{\mathrm{kt}} \mathrm{r}_{\mathrm{kt}}\right), \mathrm{k}=1,2, \ldots 10 ; \mathrm{t}=1,2, \ldots .5 \text {. }
$$

Em que k: alternativas multimodais; t: atributos do nível de serviço; e 


$$
A I_{k t}=\frac{\sum_{i=1}^{I} A I_{k i}}{\sum_{i=1}^{I} i}, \text { para todo k e t. }
$$

A importância de cada alternativa em relação a cada critério dada por cada especialista está apresentada na Tabela 3. Para todos os atributos, exceto para o tempo, a avaliação refere-se a quanto maior melhor. Para que se tivessem todos os valores dos atributos com o mesmo comportamento, o valor final da avaliação do tempo foi corrigido pela Equação 5.

$$
A I_{k 3}^{\text {corrigido }}=(1,1,1,1) \oplus(-1) \otimes A I_{k 3}
$$

0 atributo frete total da categoria custo não é uma variável qualitativa e então foi determinado de acordo com o trabalho de Leal Jr. \& D'Agosto (2011a). A Tabela 4 apresenta os seguintes valores: fretes totais para cada alternativa, as variáveis foram normalizadas de acordo com a Equação 6 e o número Fuzzy correspondente. A normalização é necessária para ter uma hierarquização em relação ao custo na escala de 0 a 1 .

$$
\text { NCusto }_{k}=\frac{\text { Custo }_{\text {menor }}}{\text { Custo }_{k}}
$$

\begin{tabular}{|c|c|c|c|c|c|c|c|c|c|c|c|c|c|c|c|}
\hline \multirow{2}{*}{ Alternativa } & \multicolumn{15}{|c|}{ Atributos } \\
\hline & & \multicolumn{2}{|c|}{ Segurança } & \multicolumn{3}{|c|}{ Flexibilidade } & & \multicolumn{2}{|c|}{ Tempo } & \multicolumn{3}{|c|}{ Capacidade } & \multicolumn{2}{|c|}{ Confiabilidade } & \\
\hline A1 & M & M & B & B & MB & B & B & MB & B & A & A & MMA & MA & A & MMA \\
\hline A2 & MMB & MB & MMB & MMA & MMA & MMA & MMA & MMA & MMA & MMA & MA & MA & A & A & MA \\
\hline A3 & MB & MB & B & B & B & B & B & B & B & MA & MA & M & B & B & M \\
\hline A4 & B & B & B & B & B & MB & B & B & $\mathrm{MB}$ & MA & MA & M & B & B & M \\
\hline A5 & MB & MB & MMB & MB & MB & M & MB & $\mathrm{MB}$ & M & A & A & A & MB & MB & B \\
\hline A6 & MB & MB & B & MB & MB & M & MB & MB & M & A & A & A & MB & $\mathrm{MB}$ & B \\
\hline A7 & B & B & B & MB & MB & M & MB & MB & M & A & A & M & MB & MB & B \\
\hline A8 & B & B & MMB & MB & MB & M & MB & MB & M & A & A & M & MB & MB & B \\
\hline A9 & B & B & MB & B & B & B & B & B & B & MA & A & M & B & B & M \\
\hline A10 & B & B & M & B & B & M & B & B & M & MA & MA & M & B & B & M \\
\hline
\end{tabular}

Tabela 3 - Avaliação de cada alternativa em relação a cada atributo para cada especialista (fonte: autores).

Tabela 4 - Frete total e números Fuzzy correspondente para cada alternativa (fonte: autores).

\begin{tabular}{lllllllllll}
\hline \multirow{2}{*}{ Alternativa } & \multirow{2}{*}{ Frete total (R\$) } & \multirow{2}{*}{ NCusto } & \multicolumn{3}{c}{ Número Fuzzy (FCk) } & \multicolumn{3}{c}{ Nível de serviço(NSk) } \\
& & & $\mathrm{q}$ & $\mathrm{o}$ & $\mathrm{p}$ & $\mathrm{r}$ & $\mathrm{q}$ & $\mathrm{o}$ & $\mathrm{p}$ & $\mathrm{r}$ \\
\hline A1 & 77,84 & 0,91 & 0,91 & 0,91 & 0,91 & 0,91 & 0,18 & 0,38 & 0,41 & 0,62 \\
$\mathrm{~A} 2$ & 97,96 & 0,72 & 0,72 & 0,72 & 0,72 & 0,72 & 0,20 & 0,41 & 0,45 & 0,66 \\
A3 & 95,54 & 0,74 & 0,74 & 0,74 & 0,74 & 0,74 & 0,10 & 0,26 & 0,27 & 0,49 \\
A4 & 92,28 & 0,76 & 0,76 & 0,76 & 0,76 & 0,76 & 0,11 & 0,28 & 0,29 & 0,50 \\
A5 & 70,52 & 1,00 & 1,00 & 1,00 & 1,00 & 1,00 & 0,09 & 0,24 & 0,25 & 0,45 \\
A6 & 83,04 & 0,85 & 0,85 & 0,85 & 0,85 & 0,85 & 0,08 & 0,24 & 0,25 & 0,46 \\
A7 & 92,39 & 0,76 & 0,76 & 0,76 & 0,76 & 0,76 & 0,07 & 0,23 & 0,24 & 0,44 \\
A8 & 95,64 & 0,74 & 0,74 & 0,74 & 0,74 & 0,74 & 0,07 & 0,22 & 0,23 & 0,43 \\
A9 & 90,11 & 0,78 & 0,78 & 0,78 & 0,78 & 0,78 & 0,11 & 0,27 & 0,29 & 0,50 \\
A10 & 99,57 & 0,71 & 0,71 & 0,71 & 0,71 & 0,71 & 0,11 & 0,28 & 0,29 & 0,51 \\
\hline
\end{tabular}

\subsection{Agregação das avaliações de cada alternativa}

A primeira agregação necessária é em relação ao nível de serviço e será empregada a Equação 7 que foi trabalhada no artigo de Liang \& Wang (1991). A Tabela 5 apresenta os valores finais da agregação (NSk).

$$
N S_{k}=\left(\frac{1}{T}\right) \otimes\left[\left(A I_{k 1} \otimes A_{1}\right) \oplus\left(A I_{k 2} \otimes A_{2}\right) \oplus \ldots \oplus\left(A I_{k T} \otimes A_{T}\right)\right]
$$

Em que T: número de atributos, 5 . A segunda agregação refere-se às categorias de custo e nível de serviço e foi utilizada a Equação 8. Os valores do indicador final (IFk) referente a agregação final para cada alternativa, são obtidos com (8):

$$
I F_{k}=\left(\frac{1}{2}\right) \otimes\left[\left(F C_{k} \otimes S_{1}\right) \oplus\left(N S_{k} \otimes S_{2}\right)\right]
$$

\subsection{Determinação de um índice para cada alternativa}

Com objetivo de verificar a hierarquização baseada nos critérios adotados ao longo do trabalho foi utilizado um indicador Fuzzy (Ik), conforme aplicado em Liang \& Wang (1991). A Equação 9 apresenta expressão utilizada para determinação do índice. Dado um conjunto de números Fuzzy Fk = (Yk, Qk, Rk, Zk), a hierarquização desses números é dada pela seguinte equação:

$$
I_{k}=\left[\frac{\left(Z_{i}-x_{1}\right)}{\left(x_{2}-x_{1}\right)-\left(R_{i}-Z_{i}\right)}+1-\frac{\left(x_{2}-Y_{1}\right)}{\left(x_{2}-x_{1}\right)+\left(Q_{i}-Y_{i}\right)}\right]^{1 / 2}
$$

Em que x1: é o mínimo do conjunto Fi; x2: é o máximo do conjunto Fi; Ik: números Fuzzy a serem comparados.

\subsection{Análise de sensibilidade}

Da hierarquização apresentada na Tabela 5, verificou-se que a alternativa 2 (A2) está representada em 4 o lugar empatada com a alternativa 9 (A9), porém a alternativa 2 é o cenário atual de transporte do bioetanol onde é utilizado basicamente o modo rodoviário. Com objetivo de tentar buscar possíveis situações onde a A2 melhorasse o desempenho de seus indicadores, decidiu-se realizar uma análise de sensibilidade, apresentando cenários variando os pesos entre as categorias do nível de serviço e custo. Além dos cenários citados, avaliou-se o desempenho isoladamente em relação a cada categoria. Para determinação dos índices foi utilizada a Equação 9 apresentada no Passo 7 do procedimento exposto neste trabalho. A Tabela 5 apresenta os resultados dos cenários da análise de sensibilidade.

\subsection{Resultado e Discussão}

A hierarquização realizada de acordo com as análises dos especialistas não apresentou a realidade logística do bioetanol, o resultado mostrou a preferência revelada pelos especialistas neste panorama. 0 cenário atual do bioetanol ficou disposto na quarta posição deste trabalho. Apesar disso, este resultado não invalida o procedimento proposto no artigo, pois não 
necessariamente a realidade é a melhor configuração ou a situação ótima. Para tanto, observou-se uma sensibilidade do desempenho das alternativas multimodais em relação à importância dada entre as categorias do nível de serviço e do custo. A Tabela 5 mostra a variação das alternativas A1, A2 e A5, apresentando cenários em relação às importâncias entre as categorias. Quando o cenário apresenta a categoria custo com maior importância em relação ao nível de serviço, a alternativa A5 mostra-se como a melhor opção. No entanto, quando os pesos das categorias se invertem, percebe-se que a alternativa A2 se apresenta como a melhor opção. Dessa forma, temos que a alternativa de equilíbrio dentre os cenários continua sendo a alternativa 1, onde apresenta maior representatividade o sistema de dutos. 0 modelo atual de transporte, a alternativa 2, é apresentada com a melhor opção apenas quando a categoria nível de serviço tem maior importância do que a categoria custo, o que leva a concluir que a configuração atual no Brasil para alternativas de transporte do bioetanol não é considerada a ótima pelos especialistas e verificada pela hierarquização através da lógica Fuzzy.

Tabela 5 - Análise de sensibilidade (fonte: autores). Legenda: NS = nível de serviço.

\begin{tabular}{|c|c|c|c|c|c|c|c|c|c|c|c|c|c|c|}
\hline \multirow{4}{*}{ Alternativa } & \multirow[b]{4}{*}{$\mathrm{Ik}$} & \multirow{4}{*}{ 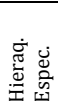 } & \multicolumn{12}{|c|}{ Cenários: Diferentes pesos entre o custo e o nível de servico } \\
\hline & & & Custo & 1 & Custo & 0 & Custo & II & Custo & I & Custo & PI & Custo & MPI \\
\hline & & & NS & 0 & NS & 1 & NS & II & NS & II & NS & II & NS & \\
\hline & & & $\mathrm{Ik}$ & Hieraq. & $\mathrm{Ik}$ & Hieraq. & $\mathrm{Ik}$ & Hieraq. & $\mathrm{Ik}$ & Hieraq. & $\mathrm{Ik}$ & Hieraq. & $\mathrm{Ik}$ & Hieraq \\
\hline A1 & 0,60 & 2 & 0,68 & 2 & 0,54 & 2 & 0,63 & 1 & 0,55 & 1 & 0,53 & 1 & 0,50 & 2 \\
\hline A2 & 0,45 & 4 & 0,04 & 9 & 0,58 & 1 & 0,52 & 3 & 0,50 & 2 & 0,52 & 2 & 0,53 & 1 \\
\hline A3 & 0,40 & 8 & 0,10 & 7 & 0,39 & 6 & 0,41 & 7 & 0,40 & 8 & 0,41 & 8 & 0,38 & 6 \\
\hline $\mathrm{A} 4$ & 0,43 & 6 & 0,19 & 5 & 0,41 & 4 & 0,44 & 6 & 0,42 & 6 & 0,43 & 5 & 0,40 & 3 \\
\hline A5 & 0,63 & 1 & 1,00 & 1 & 0,35 & 8 & 0,59 & 2 & 0,48 & 3 & 0,46 & 3 & 0,37 & 7 \\
\hline A6 & 0,49 & 3 & 0,48 & 3 & 0,36 & 7 & 0,48 & 4 & 0,43 & 4 & 0,42 & 6 & 0,37 & 8 \\
\hline A7 & 0,40 & 7 & 0,19 & 6 & 0,34 & 9 & 0,40 & 9 & 0,39 & 9 & 0,39 & 9 & 0,35 & 9 \\
\hline A8 & 0,37 & 10 & 0,10 & 8 & 0,33 & 10 & 0,38 & 10 & 0,37 & 10 & 0,38 & 10 & 0,34 & 10 \\
\hline A9 & 0,44 & 5 & 0,25 & 4 & 0,40 & 5 & 0,45 & 5 & 0,43 & 5 & 0,43 & 4 & 0,39 & 5 \\
\hline A10 & 0,38 & 9 & 0,00 & 10 & 0,41 & 3 & 0,40 & 8 & 0,40 & 7 & 0,42 & 7 & 0,40 & 4 \\
\hline
\end{tabular}

\section{Conclusão}

A teoria de conjuntos Fuzzy foi capaz de modelar o comportamento e variáveis qualitativas do problema estudado neste trabalho. Porém, da visualização de um único cenário para a hierarquização das alternativas não foi considerada como satisfatória. A análise de sensibilidade realizada no trabalho mostrou comportamentos mais detalhados das variáveis e gerou informações pertinentes para auxiliar a tomada de decisão em ambientes de escolha de alternativas de transportes. 0 aumento do peso do nível de serviço em relação ao custo fez com que esta alternativa melhorasse o desempenho e fosse a melhor das alternativas. A análise de sensibilidade mostrou que o nível de serviço pode ser um item de maior importância do que o custo para explicar a preferência revelada das alternativas, porém cabe avaliar que o valor aparente do nível de serviço em relação ao custo pode estar distorcido devido à falta de infraestrutura para os demais modais de transporte impossibilitando a viabilidade de alternativas com melhor desempenho. Como proposta de estudos futuros, tem-se a possibilidade de avaliar outros produtos com alternativas diferentes e com diferentes grupos de especialistas para avaliar a influência do nível do especialista na resposta do problema.

\section{Referências}

Acosta, C. M. M., Silva, A. M. V. A., \& Lima, M. L. P. (2011). Aplicação de análise envoltória de dados (DEA) para medir eficiência em portos brasileiros. Journal of Transport Literature, 5(4), 88-102.

Bertoloto, R. F., \& Soares de Mello, J. C. C. B. (2011). Eficiência de portos e terminais privativos brasileiros com características distintas. Journal of Transport Literature, 5(2), 4-21.

Ciarlini, M., \& Correia, A. R. (2011). Aplicação de método multicritério no planejamento de sistema de transporte rápido de massa. Journal of Transport Literature, 5(3), 192-209.

Cortez, L. C. S., Oliveira, L. R., Martins, E. F., Jesus, I. R. D., \& Mello, J. C. C. B. S. (2013). Análise de eficiência na gestão de portos públicos brasileiros em relação ao papel das autoridades portuárias. Journal of Transport Literature, 7(2), 78-96.

Gabriele, P. D., Brandão, L. C., Treinta, F. T., Mello, J. C. C. B. S., \& Carvalhal, R. (2013). Comparação internacional da eficiência ambiental dos modos de transporte rodoviário e ferroviário. Journal of Transport Literature, 7(1), 212-229.

Guabiroba R. C. S., D'Agosto, M. A., Leal Junior, I. C., \& Vieira da Silva, M. A. (2014). Eco-efficiency as an auxiliary measure for the definition of interregional public consortia responsible for the collection of recyclable domestic waste. Journal of Cleaner Production , 68, 36-45.

Falcão, V. A., \& Correia, A. R. (2012). Eficiência portuária: análise das principais metodologias para o caso dos portos brasileiros. Journal of Transport Literature, 6(4), 133-146.

Fernandes, E., \& Pires, H. M. (2011). Desempenho de empresas aéreas: uma análise mundial. Journal of Transport Literature, 5(3), 123-140.

Leal Jr, I. C. (2010). Método de Escolha Modal para transporte de produtos perigosos com base em medidas de ecoeficiência. Tese de doutorado. UFRJ/COPPE, Rio de Janeiro.

Leal Jr., I.C., \& D'Agosto, M. de A. (2011a). Modal choice for transportation of hazardous materials: the case of land modes of transport of bio-ethanol in Brazil. Journal of Cleaner Production, 19, 229-240.

Leal Jr., I.C., \& D'Agosto, M. de A. (2011b). Modal choice evaluation of transport alternatives for exporting bio-ethanol from Brazil. Transportation Research Part D, 16, 201-207.

Liang, G., \& Wang, M. J. (1991). A fuzzy multi-criteria decision-making method for facility site selection. Int. J. Prod. Res., 29(11), 2313-2330.

Lieggio Junior, M., Granemann, S. R. and Souza, O. A. (2012) Aplicabilidades da análise multicritério às problemáticas de decisão no transporte rodoviário de produtos perigosos: uma perspectiva teórica. Journal of Transport Literature, vol. 6, n. 2, pp. $197-217$.

\section{Abstract}

This paper presents a procedure to assist the performance analysis of transport alternatives with the combined use of quantitative and linguistic variables, differing from the traditional assessment that use only quantitative variables in their applications. The proposed procedure employs fuzzy logic to model the linguistic variables that represent the level of service of the alternatives and the weights of the attributes of the problem. The procedure was applied for a bioethanol transportation case for which we evaluated the cost categories and the level of service of the available multimodal alternatives from the perspective of field experts. We implemented a sensitivity analysis through scenarios with variations in the importance of these categories. Results show that the assessment of experts there is a tendency to assign greater importance to the level of service compared to the cost of the operation and that the current practice of transport is not the most appropriate alternative considering the views of experts.

Key words: fuzzy logic, bioethanol, modal choice. 